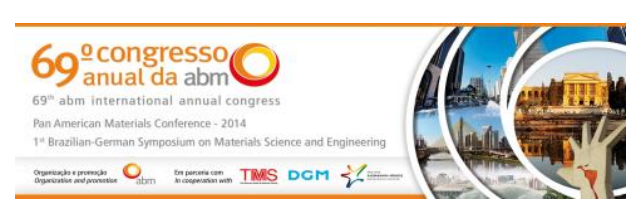

Tema: Diagramas de fases e transformações de fases

\title{
ESTUDO DA ESCÓRIA ESPUMANTE EM FORNO ELÉTRICO A ARCO*
}

\author{
Mateus Alves Segundo Paulino \\ Jeferson Leandro Klug ${ }^{2}$ \\ Wagner Viana Bielefeldt ${ }^{3}$ \\ Antônio Cezar Faria Vilela 4 \\ Nestor Cezar Heck
}

\section{Resumo}

A espumação de escória pode ser definida como sendo o aumento na espessura da camada de escória que ocorre devido à evolução de bolhas de $\mathrm{CO}(\mathrm{g})$ durante a operação do forno elétrico a arco. Um dos maiores benefícios obtidos com a espumação da escória no forno elétrico a arco é a diminuição dos custos com eletricidade. O presente trabalho tem por objetivos estudar por meio da termodinâmica computacional alguns tópicos do sistema quaternário $\mathrm{CaO}-\mathrm{SiO}_{2}-\mathrm{FeO}-\mathrm{MgO}$ (CSFM) importante na siderurgia pela conexão com o tema espumação de escória, que são: (i) composições de saturação das fases sólidas 'MgO' e 'CaO' e (ii) a viscosidade efetiva de um ponto particular deste sistema.

Palavras-chave: Escória espumante; FEA; Dupla saturação.

\begin{abstract}
STUDY OF FOAMING SLAG IN ELECTRIC ARC FURNACE
Abstract

Foaming slag can be defined as the increase in thickness of the slag layer which occurs due to the evolution of $\mathrm{CO}(\mathrm{g})$ bubbles during operation of the electric arc furnace. One of the biggest benefits derived from slag foaming in the electric arc furnace is the reduction of electricity costs. The present work aims to study through computational thermodynamics some topics of the quaternary system $\mathrm{CaO}-\mathrm{SiO}_{2}-\mathrm{FeO}-\mathrm{MgO}$ (CSFM) important in steelmaking in connection with the theme foaming slag, which are: (i) saturation compositions of the solid phases 'MgO' and ' $\mathrm{CaO}$ ' and (ii) the effective viscosity of a particular point of this system.
\end{abstract}

Keywords: Foaming slag; EAF; Double saturation

Eng. Met., Depart. de Engenharia Metalúrgica e de Materiais, PPGECM, UFC, Fortaleza, Brasil. Eng. Met., Dr., Depart. de Eng. Metalúrgica e de Materiais, PPGECM, UFC, Fortaleza, Brasil.

Eng. Met., Dr., Depart. de Eng. Metalúrgica, LASID, PPGE3M, UFRGS, Porto Alegre, RS, Brasil.

Eng. Met., Dr., Depart. de Eng. Metalúrgica, LASID, PPGE3M, UFRGS, Porto Alegre, RS, Brasil.

Eng. Met., Dr., Depart. de Eng. Metalúrgica, NTCm, PPGE3M, UFRGS, Porto Alegre, RS, Brasil.

\footnotetext{
* Contribuição técnica ao 69 Congresso Anual da ABM - Internacional e ao 14ํㅡㄹ ENEMET - Encontro Nacional de Estudantes de Engenharia Metalúrgica, de Materiais e de Minas, 21 a 25 de julho de 2014, São Paulo, SP, Brasil.
} 


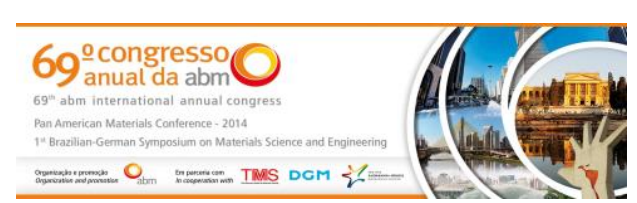

\section{INTRODUÇÃO}

\subsection{O FEA e a Escória Espumante}

A demanda pelo material aço não exime seus produtores de examinarem continuamente os processos produtivos, para que um volume adequado deste material seja colocado no mercado ao menor custo possível de produção.

Um grande número de produtores de aço se utiliza do forno elétrico a arco, FEA, para a produção deste material. Um FEA deve combinar alta capacidade com máxima disponibilidade, e o processamento de uma variedade de materiais de carga com a produção de uma boa gama de tipos de aço. Ao mesmo tempo, são fatores importantes tanto a redução dos custos operacionais quanto a limitação do impacto sobre o meio ambiente.

Considerando-se os custos de operação de um forno elétrico a arco, os seguintes tópicos e a correspondente fração do custo total podem ser considerados, Tabela 1; verifica-se pela Tabela, que o custo com eletricidade é o segundo parâmetro em ordem de importância [1].

Tabela 1. Custos relativos na operação de um forno elétrico a arco [1]

\begin{tabular}{|l|c|}
\hline Parâmetro & Custo típico [\%] \\
\hline Carga do forno & 71,0 \\
\hline Eletricidade & 14,2 \\
\hline Eletrodos & 11,3 \\
\hline Mão-de-obra & 2,1 \\
\hline Refratários & 1,4 \\
\hline
\end{tabular}

No FEA, a eletricidade é usada na geração do calor necessário para a fusão da carga e a sua manutenção numa temperatura adequada. O calor é gerado por meio de arcos elétricos e a tranferência da energia se dá principalmente por radiação; para se evitar a perda de calor por radiação para a abóbada e as paredes do forno, é interessante o uso da técnica de arco submerso na escória espumante.

A espumação de escória pode ser definida como sendo o aumento na espessura da camada de escória que ocorre devido à evolução de bolhas de $\mathrm{CO}_{(\mathrm{g})}$ durante a operação do forno elétrico a arco.

Efetivamente, um dos benefícios obtidos com a escória espumante no forno elétrico a arco é a diminuição dos custos com eletricidade, mas, há outras [1-4]:

- melhor transferência de energia para o banho (economia de quantidade considerável de energia);

- proteção dos refratários de desgaste excessivo;

- redução do ruído provocado pelo arco elétrico (algumas usinas se baseiam no som do arco elétrico e em parâmetros elétricos do FEA para avaliar a qualidade da espumação);

- diminuição de perda de calor para as paredes do forno;

- diminuição da incorporação de nitrogênio pelo banho;

- aumento do comprimento de arco elétrico (fato que reduzindo o consumo de eletrodos).

\footnotetext{
* Contribuição técnica ao $69^{\circ}$ Congresso Anual da ABM - Internacional e ao 14ํㅡㄹ ENEMET - Encontro Nacional de Estudantes de Engenharia Metalúrgica, de Materiais e de Minas, 21 a 25 de julho de 2014, São Paulo, SP, Brasil.
} 

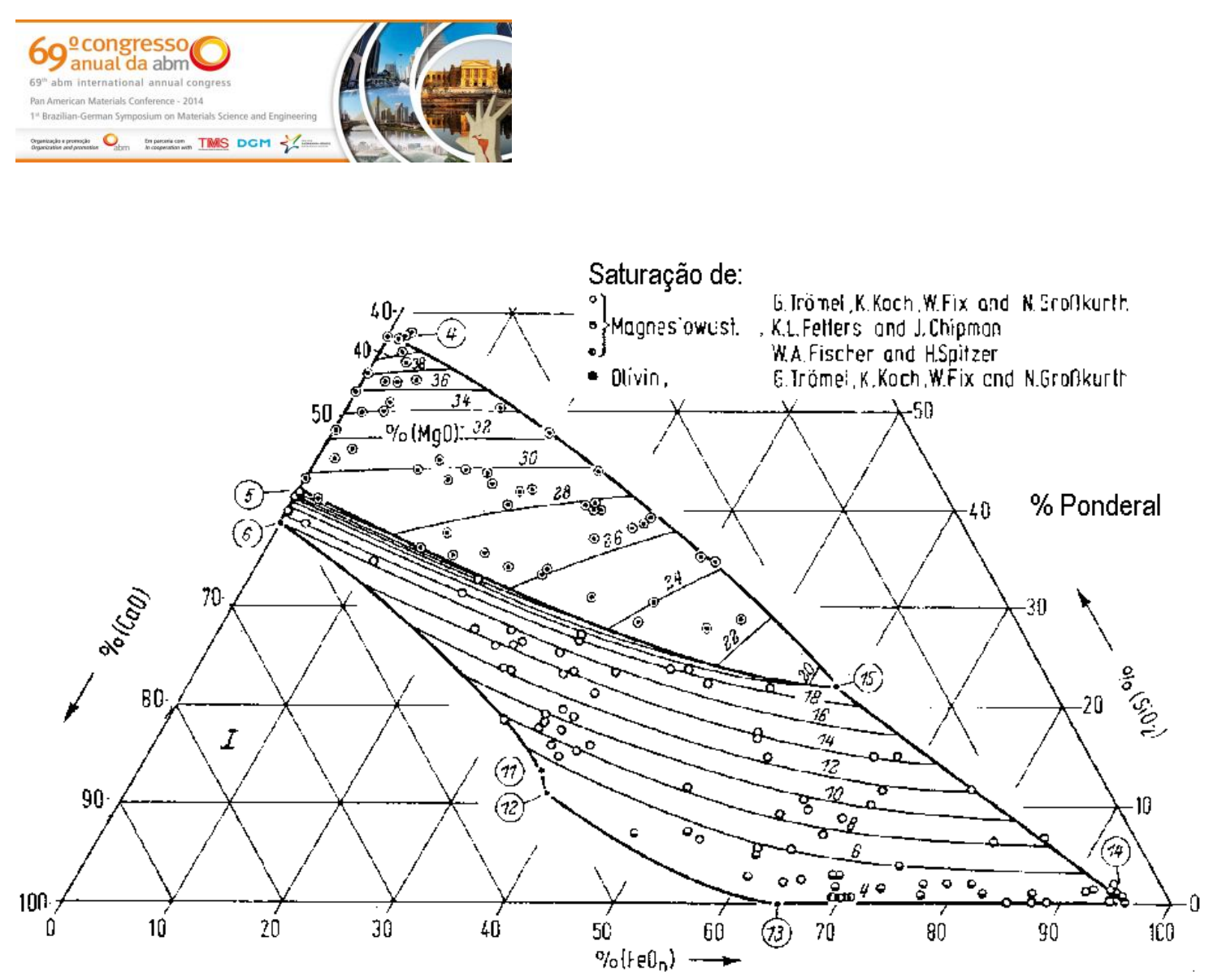

Figura 3. Projeção das superfícies $\mathrm{MgO}$. FeO e $\mathrm{MgO}$.SiO 2 no diagrama $\mathrm{CaO}-\mathrm{SiO}_{2}-\mathrm{MgO} ; \mathrm{T}=1600^{\circ} \mathrm{C}$ [13].

Uma curva relacionando o teor de $\mathrm{MgO}$ necessário para a obtensão da dupla saturação em função da basicidade foi confeccionada por Pretorius e Carlisle [2] (ver adiante).

\subsection{Diagramas Isotérmicos de Solubilidade - ISD}

Dados de solubilidade do $\mathrm{MgO}$ em função da basicidade da escória podem ser muito úteis para a a compatibilidade com os refratários, mas não oferecem uma visão clara na questão relacionada à espumação das escórias, pois o efeito do teor de $\mathrm{FeO}$ embora possa ser calculado - não é mostrado. Tendo isto em mente, aqueles autores se debruçaram novamente sobre a questão, com o objetivo apresentar um esquema mais completo.

Como durante a fusão do aço no FEA tanto a basicidade da escória (determinada basicamente pelos níveis de Si e de Al na sucata metálica e pela quantia de óxidos refratários $\mathrm{MgO}$ e $\mathrm{CaO}$ adicionados com a carga) quanto a temperatura permanecem praticamente fixas, as relações entre as fases nestas condições são aquelas que maior interesse suscitam.

Assim, para apresentar os efeitos entre $\mathrm{MgO}, \mathrm{FeO}$ e as diferentes fases do sistema, sob basicidade e temperatura constantes, Pretorius e Carlisle [2] criaram os assim chamados diagramas isotérmicos de solubilidade, abreviados por ISD na literatura de língua inglesa - um exemplo dos quais se encontra na Figura 4.

Assim sendo, para cada ISD, as variáveis são: as concentrações de $\mathrm{MgO}$ e FeO. A primeira delas representa a interação entre a escória e os refratários básicos do forno, enquanto que a segunda reproduz o grau de oxidação do sistema.

* Contribuição técnica ao $69^{\circ}$ Congresso Anual da ABM - Internacional e ao 14ํㅡㄹ ENEMET - Encontro Nacional de Estudantes de Engenharia Metalúrgica, de Materiais e de Minas, 21 a 25 de julho de 2014, São Paulo, SP, Brasil. 

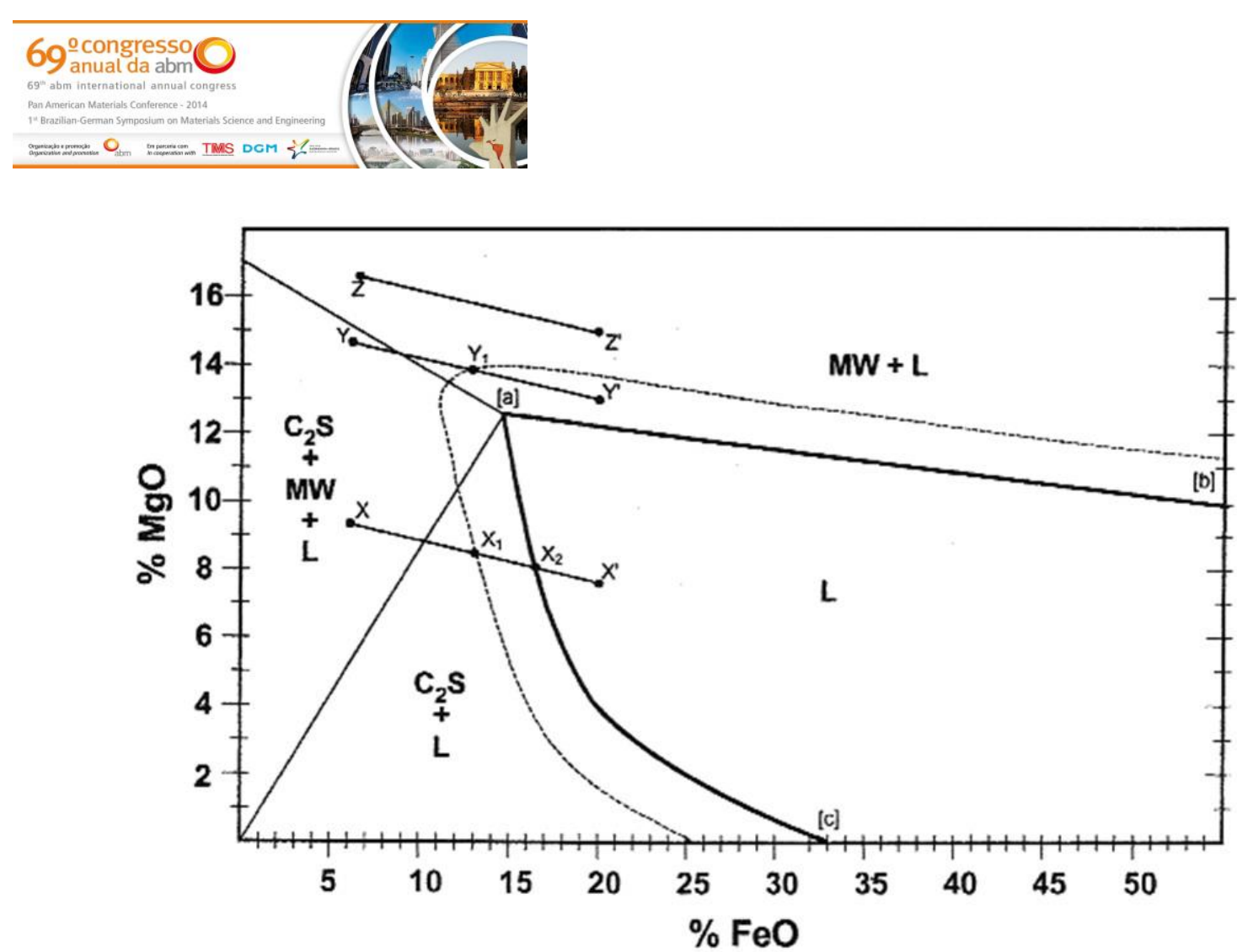

Figura 4. Diagrama Isotérmico de Solubilidade (ISD) para a basicidade ternária $B_{3}=$ igual a 1,5 à temperatura de $1600^{\circ} \mathrm{C} ;\left(\mathrm{C}=\mathrm{CaO}, \mathrm{S}=\mathrm{SiO}_{2}, \mathrm{M}=\mathrm{MgO}, \mathrm{W}=\mathrm{FeO}\right.$, e $\mathrm{L}=$ escória líquida) [2].

$\mathrm{Na}$ Figura 4, três escórias de composições diferentes - representadas pelas letras $\mathrm{X}$, Y e Z - serão usadas para auxiliar o entendimento da relação entre a composição (teores de $\mathrm{FeO}$ e de $\mathrm{MgO}$ ) e o comportamento esperado para a escória, para temperatura e basicidade mantidas constantes.

Para a escória $\mathrm{X}$, as fases secundárias consistem de $\mathrm{C}_{2} \mathrm{~S}, \mathrm{MW}$ e, provavelmente ela é muito encrostada para espumar bem nesse estágio. À medida que o conteúdo de FeO na escória aumenta (devido à injeção de oxigênio), o conteúdo de $\mathrm{MgO}$ da escória é diluído e ela segue da composição inicial X para a final X'. À medida que a composição da escória vai de $\mathrm{X}$ para $\mathrm{X}_{1}$, a viscosidade da escória muda de encrostada para fofa, e as suas características de espumação melhoram. Entretanto, enquanto a composição da escória continua se deslocando, do ponto $X_{1}$ para $X_{2}$, a viscosidade efetiva da escória diminui, na proporção em que a quantidade de líquido aumenta. Isto provoca um decréscimo na sua capacidade de espumação. No ponto $X_{2}$ a escória está totalmente líquida, e permanece assim enquanto a composição de $\mathrm{FeO}$ avança para o ponto X'. Em relação à proteção de refratários do FEA, pode-se dizer que, na composição inicial (ponto $\mathrm{X}$ ), a escória está saturada com $\mathrm{MgO}$, mas, à medida que a quantidade de FeO aumenta, a escória torna-se insaturada, tornando-se agressiva aos refratários.

Para a escória $Y$, a quantidade inicial de $F e O$ é idêntica à da escória $X$, mostrando que ela é uma escória do tipo encrostada, com características ruins de espumação (as fases precipitadas são as mesmas encontradas na escória de composição X). De $Y$ para $Y_{1}$ as características de espumação da escória melhoram. À medida que a composição da escória se desloca de $Y_{1}$ para $Y^{\prime}$, a boa qualidade de espumação se mantém, devido à presença de partículas de segunda fase MW. Isto contrasta com as escórias $\mathrm{X}_{2}$ e $\mathrm{X}_{1}$, que são totalmente líquidas a partir de certos níveis de FeO. Em relação à proteção do refratário do forno, ela é totalmente compatível com o refratário ao longo de todo o intervalo de composição (saturado em $\mathrm{MgO}$ ). Assim, a escória $\mathrm{Y}$ não somente é compatível com o refratário, mas as suas características da

\footnotetext{
* Contribuição técnica ao $69^{\circ}$ Congresso Anual da ABM - Internacional e ao 14ํㅡㄹ ENEMET - Encontro Nacional de Estudantes de Engenharia Metalúrgica, de Materiais e de Minas, 21 a 25 de julho de 2014, São Paulo, SP, Brasil.
} 

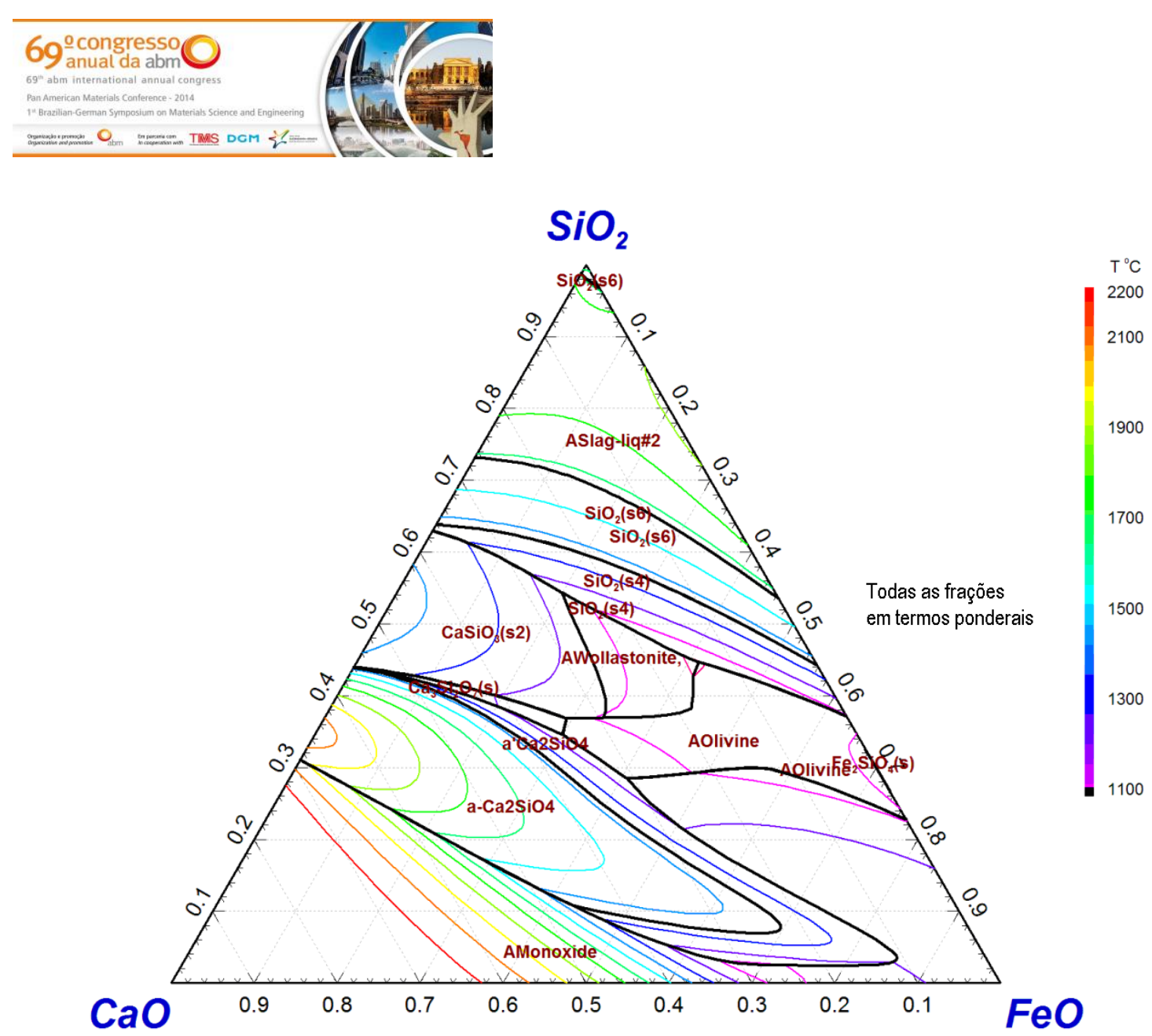

Figura 5. Projeção das superfícies liquidus do sistema $\mathrm{CaO}-\mathrm{SiO}_{2}-\mathrm{FeO}$ (calc. via FactSage)

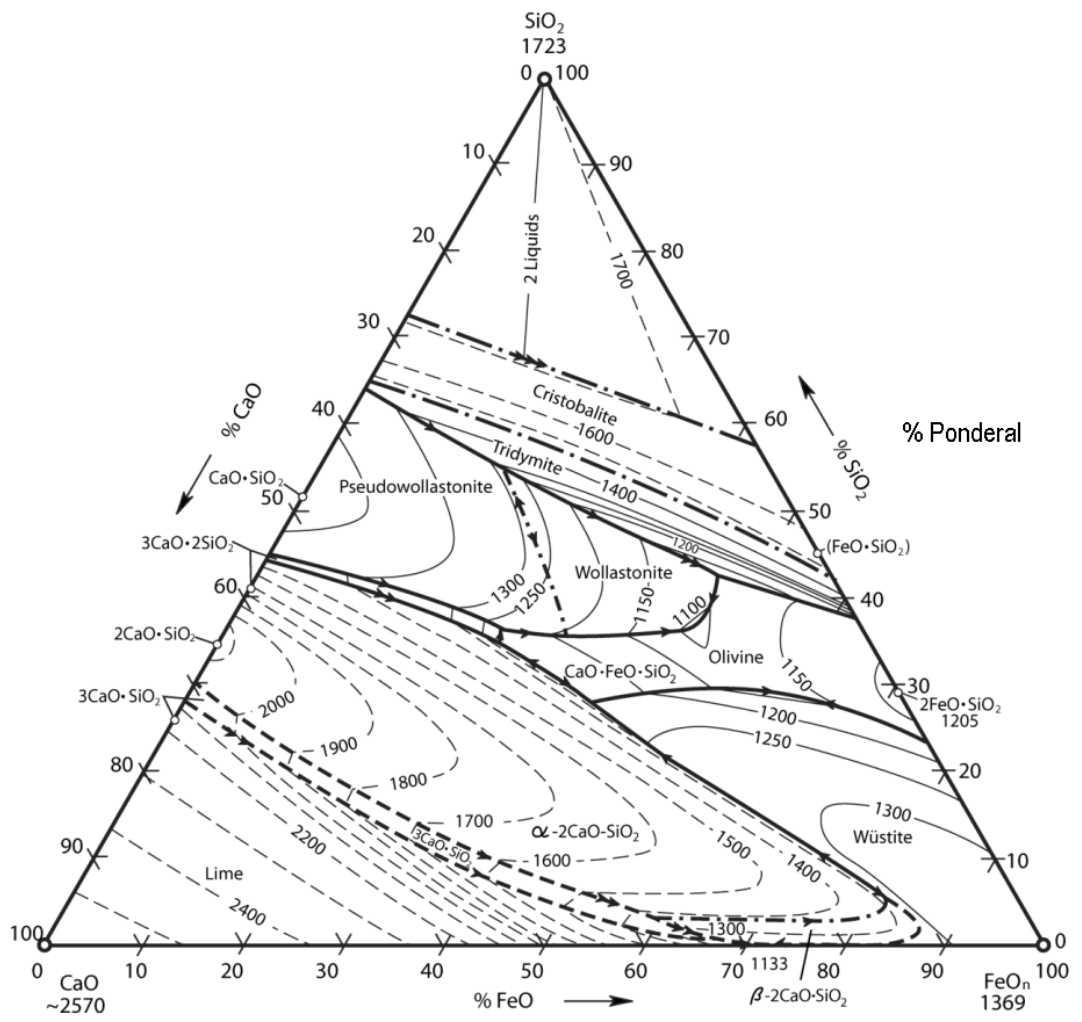

Figura 6. Projeção das superfícies liquidus do sistema $\mathrm{CaO}-\mathrm{SiO}_{2}-\mathrm{FeO}$ [13].

* Contribuição técnica ao 69ำ Congresso Anual da ABM - Internacional e ao 14ํㅡㄹ ENEMET - Encontro Nacional de Estudantes de Engenharia Metalúrgica, de Materiais e de Minas, 21 a 25 de julho de 2014, São Paulo, SP, Brasil. 
Considerando a importância do óxido MgO sobre o sistema CSF, a Figura 7 mostra o campo monofásico Líquido, no sistema CSFM, para 5\% de MgO.

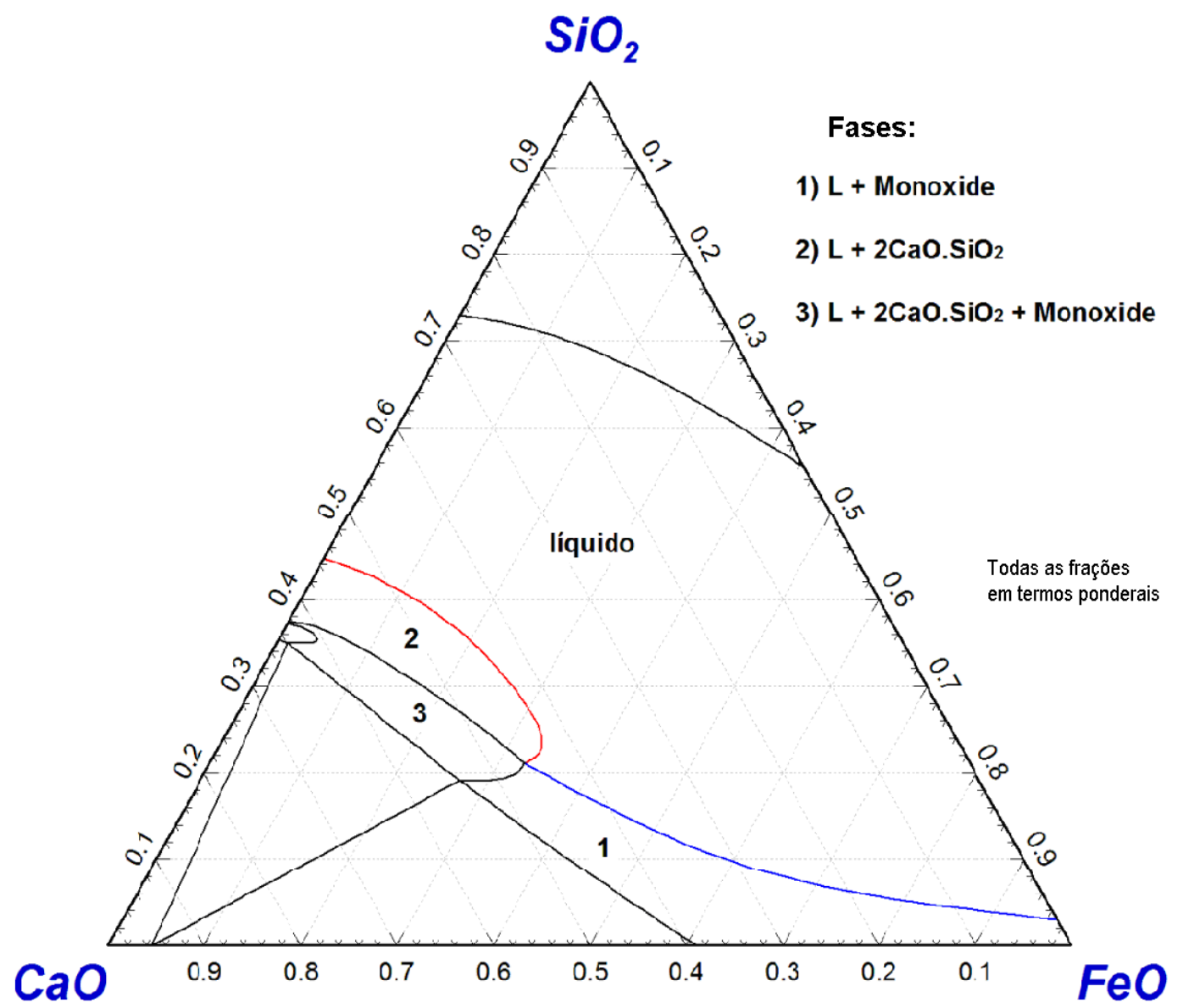

Figura 7. Corte isotérmico no diagrama CSFM para $5 \%$ de $\mathrm{MgO} ; \mathrm{T}=1600^{\circ} \mathrm{C}$ (calculado com o FactSage)

Nesta figura também são dados destaques para outros 3 campos:

1. Líquido + Monoxide (solução sólida magnésio-wustita);

2. Líquido + $2 \mathrm{CaO} \cdot \mathrm{SiO}_{2}$;

3. Líquido + Monoxide + $2 \mathrm{CaO} . \mathrm{SiO}_{2}$.

O ponto de intersecção dos três campos citados acima pode ser chamado de ponto de dupla saturação - neste caso, saturação em ' $\mathrm{CaO}$ ' (2CaO.SiO 2$)$ e ' $\mathrm{MgO}$ ' (Monoxide). A composição deste ponto no diagrama fornece a basicidade binária e o teor de $\mathrm{FeO}$ em equilíbrio, para o teor de $5 \%$ de $\mathrm{MgO}$. É possível repetir o mesmo cálculo para outros teores de $\mathrm{MgO}$ (ver adiante).

O Slag Atlas fornece a projeção das diversas superfícies encontradas no sistema CSFM para a temperatura de $1600^{\circ} \mathrm{C}$ no diagrama ternário $\mathrm{CaO}-\mathrm{SiO}_{2}-\mathrm{FeO}$ (base da representação espacial do sistema já citado). Os resultados estão fundamentados nos trabalhos de Trömmel et al. [22] e Görl et al. [23]. As projeções também apresentam curvas de isoconcentração de MgO.

\subsection{Teor de MgO para a Dupla Saturação}

Conforme já foi visto, as superfícies de precipitação das fases sólidas 'CaO' (2CaO.SiO2) e 'MgO' (MgO.FeO) têm uma curva em comum no sistema CSFM. As

* Contribuição técnica ao $69^{\circ}$ Congresso Anual da ABM - Internacional e ao 14ํㅡㄹ ENEMET - Encontro Nacional de Estudantes de Engenharia Metalúrgica, de Materiais e de Minas, 21 a 25 de julho de 2014, São Paulo, SP, Brasil. 


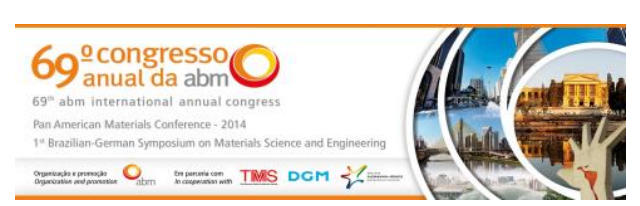

composições descritas por essa curva terão, ao mesmo tempo, saturação em 'MgO' e 'CaO'. Este fato é de interesse prático, por causa da proteção aos refratários do forno e pela possibilidade de serem atingidos valores altos no índice de espumação. A Figura 8 mostra os teores de MgO necessários para a dupla saturação de uma escória do sistema CSFM, como função de sua basicidade binária (\%CaO/\% $\left.\mathrm{SiO}_{2}\right)$, obtidos via simulação (Figura 3), em conjunto com os dados apresentados por Pretorius e Carlisle [2].

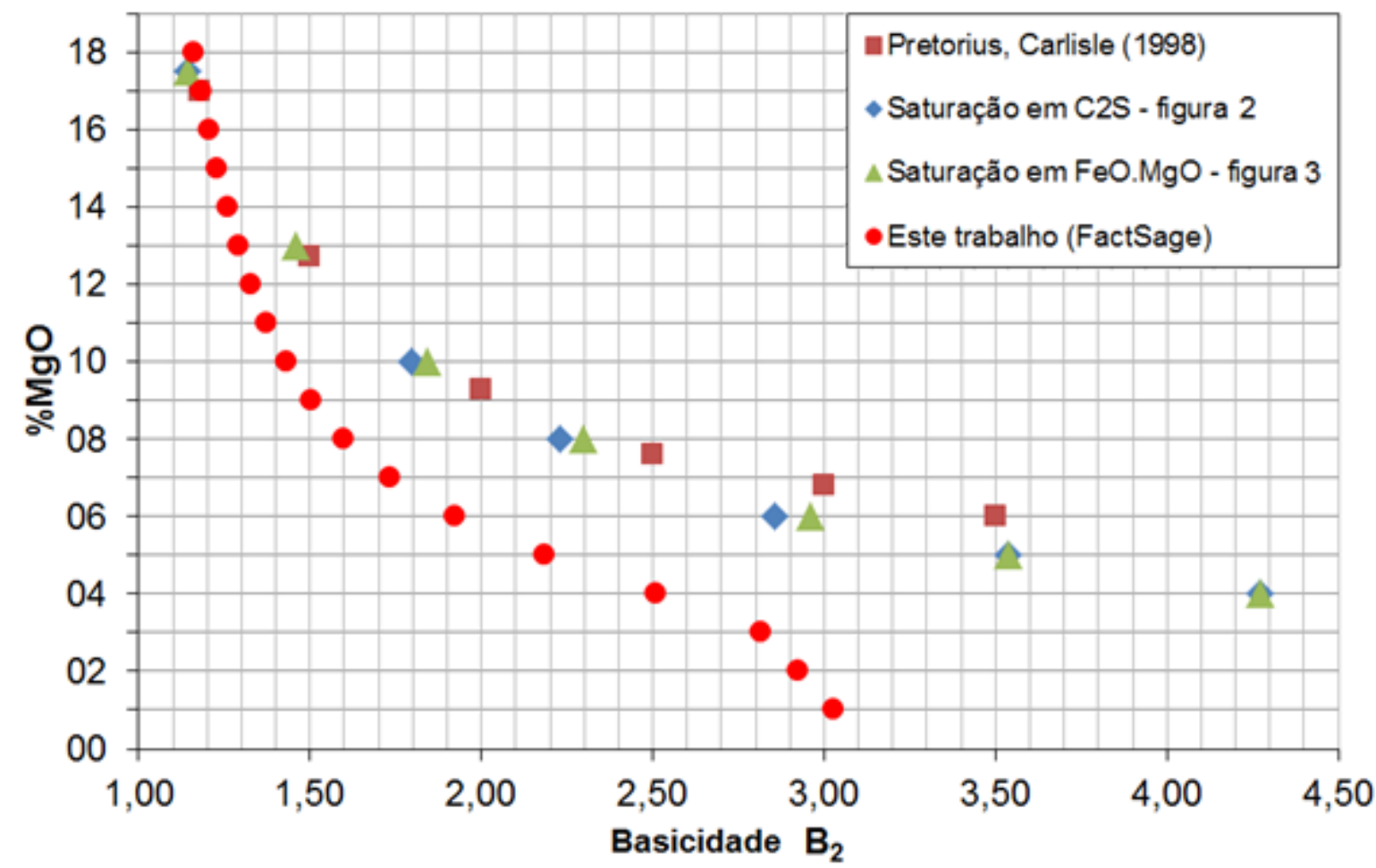

Figura 8. Teor de MgO necessário para a dupla saturação de escórias do sistema CSFM, como função da basicidade $\% \mathrm{CaO} / \% \mathrm{SiO}_{2} ; \mathrm{T}=1600^{\circ} \mathrm{C}$

Na Figura 8 é visível a diferença entre as curvas de Pretorius e Carlisle, as curvas provindas das Figuras 2 e 3, de Trömmel et ali. [22] e Görl et ali. [23], e os resultados deste trabalho. A diferença fica em torno de $3 \%$ de $\mathrm{MgO}$ entre basicidade 1,4 e 2,8; a partir de $\mathrm{B}_{2}=2,8$ a curva calculada sofre forte queda. É provável que, para valores maiores do que 3,1, ela não intercepta mais os pontos de dupla saturação

As curvas de Pretorius e Carlisle e as provindas das Figuras 2 e 3 têm boa concordância entre si, pois as figuras citadas serviram de base para o trabalho de Pretorius e Carlisle. No presente trabalho não foi levado em consideração o efeito da pressão parcial de oxigênio na oxidação do ferro. Bennett e Kwong [24], contudo, realizaram um estudo empregando a termodinâmica computacional (FactSage) para avaliar o sistema CSFM e obtiveram resultados consistentes com os da literatura.

\subsection{Viscosidade Efetiva}

A Figura 9 reproduz o ISD para uma escória com basicidade $\mathrm{B}_{3}=2,0$ à temperatura de $1600^{\circ} \mathrm{C}$ (o índice $\mathrm{B}_{3}$ também considera o teor de $\mathrm{Al}_{2} \mathrm{O}_{3}$ na equação da basicidade).

\footnotetext{
* Contribuição técnica ao 69ำ Congresso Anual da ABM - Internacional e ao 14ํㅡㄹ ENEMET - Encontro Nacional de Estudantes de Engenharia Metalúrgica, de Materiais e de Minas, 21 a 25 de julho de 2014, São Paulo, SP, Brasil.
} 


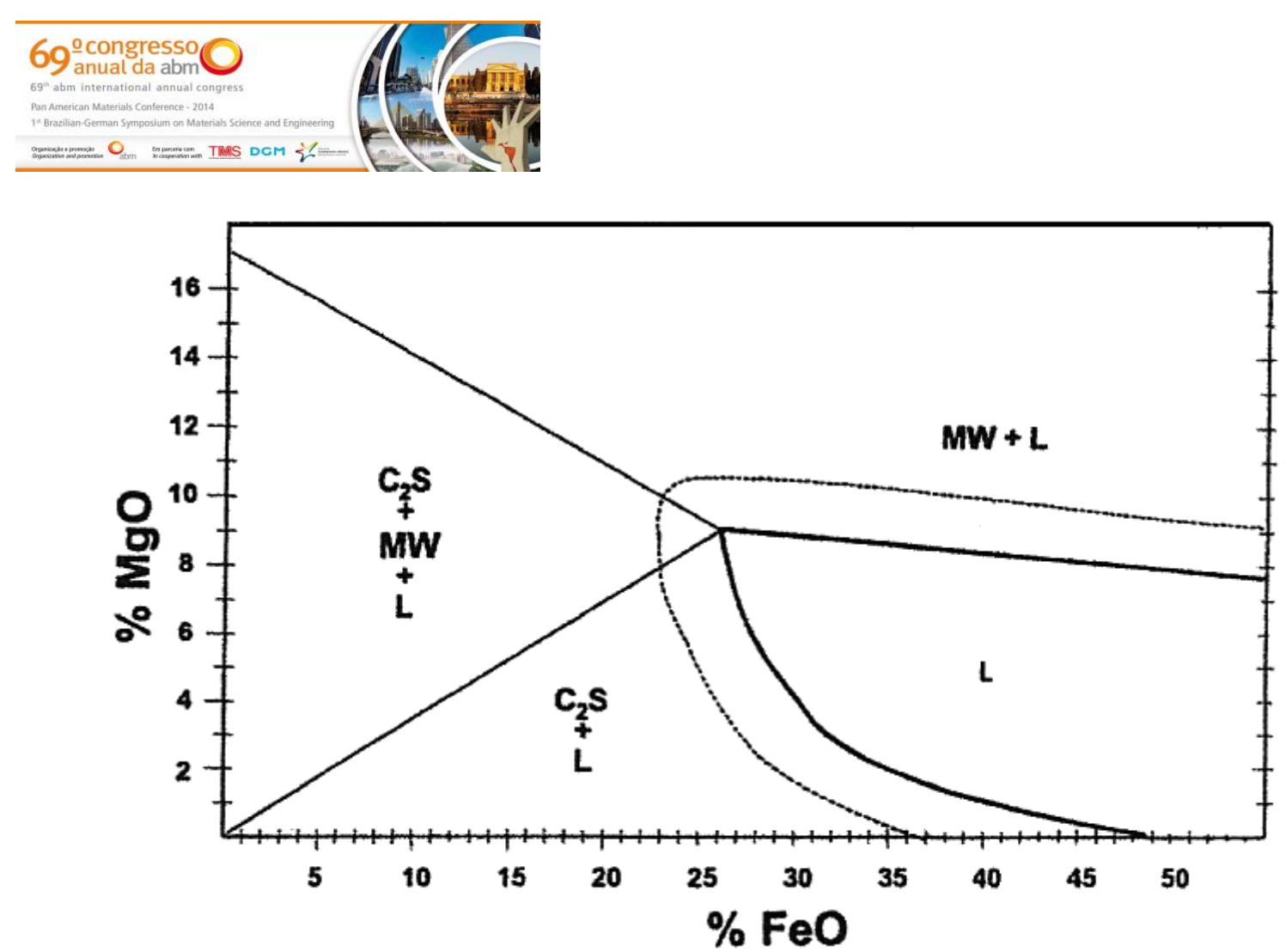

Figura 9. Diagrama Isotérmico de Solubilidade (ISD) para a basicidade $\mathrm{B}_{3}=2,0\left(\mathrm{C}_{2} \mathrm{~S}=2 \mathrm{CaO} . \mathrm{SiO}_{2}\right.$, $\mathrm{MW}=$ Magnésio-Wustita, e L = Líquido) [2].

A área considerada ideal para a espumação por Pretorius e Carlisle é aquela entre o campo totalmente líquido e o delimitado pela linha pontilhada, contendo as fases líquido mais magnésio-wustita (MW). Na Figura 9, entre aproximadamente entre 8 e $10 \%$ de $\mathrm{MgO}$ e 25 a $50 \%$ de $\mathrm{FeO}$, tem-se uma escória líquida saturada em $\mathrm{MgO}$ (MW) com uma fração de sólidos apenas suficiente para a nucleação de bolhas de CO. Nela, mesmo com a variação no teor de $\mathrm{FeO}$ no decorrer do processo, seria possível manter as boas condições de espumação e a compatibilidade com os refratários à base de $\mathrm{MgO}$.

Uma investigação sobre a variação da fração de fase líquida e das fases sólidas obtidas por meio do software FactSage foi feita partindo-se da composição $10 \%$ de $\mathrm{MgO}$ e um pequeno teor inicial de $\mathrm{FeO}$, escolhido de modo que o sistema esteja dentro do campo ternário $C_{2} S+M W+L$. Outras condições são: $B_{2}=2,0$ e $T=1600^{\circ} \mathrm{C}$. $O$ resultado dessa simulação pode ser visto na Figura 10.

A Figura 10 mostra que a fração de líquido aumenta com o aumento do teor de $\mathrm{FeO}$ - o que era esperado. Somente a partir de $20 \%$ de $\mathrm{FeO}$ é que a fração de líquido supera a fração de sólidos.

Para a descrição da viscosidade de líquidos contendo partículas de segunda fase, a equação de Roscoe-Einstein pode ser utilizada [14]:

$$
\eta_{e}=\eta(1-\rho \Theta)^{-5 / 2}
$$

Onde:

$\eta_{e} \quad$ viscosidade efetiva da escória;

$\eta \quad$ viscosidade da escória líquida;

$\Theta \quad$ fração de fases sólidas (precipitados);

$\rho$ parâmetro de interação do sólido.

* Contribuição técnica ao 69ำ Congresso Anual da ABM - Internacional e ao 14ํㅡㄹ ENEMET - Encontro Nacional de Estudantes de Engenharia Metalúrgica, de Materiais e de Minas, 21 a 25 de julho de 2014, São Paulo, SP, Brasil. 


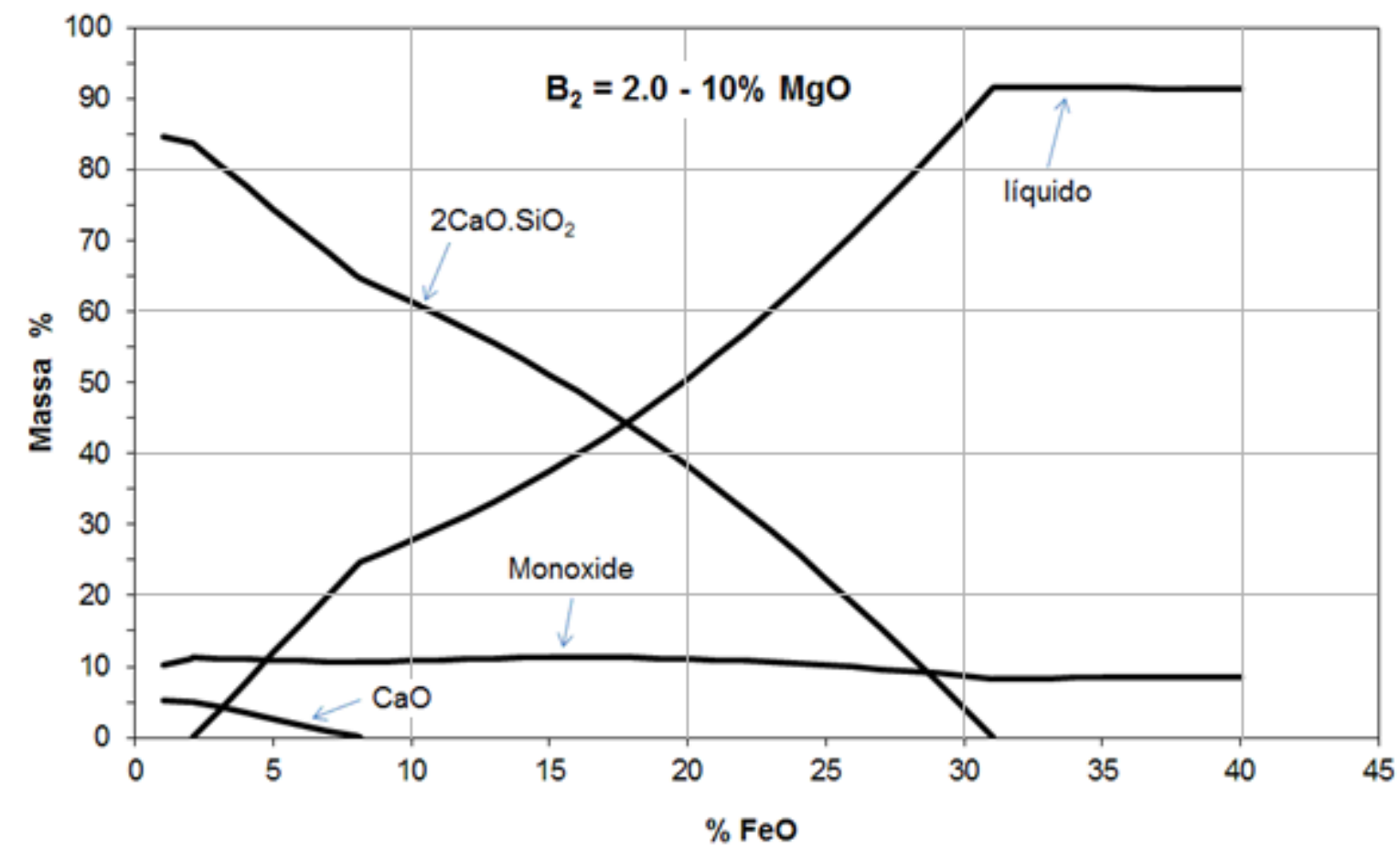

Figura 10. Massa das fases em equilíbrio em função do teor de $\mathrm{FeO}$ em escórias; $\mathrm{B}_{2}=2,0, \mathrm{MgO}$ $=10 \%$ e $\mathrm{T}=1600^{\circ} \mathrm{C}$ (Calculado com o FactSage)

Para uma suspensão diluída de esferas de diferentes tamanhos, o parâmetro de interação do sólido é igual à unidade - valor utilizado neste trabalho.

Tabela 2. Viscosidade da escória líquida e viscosidade efetiva; $\mathrm{B}_{2}=2,0, \mathrm{MgO}=10 \%$ e $\mathrm{T}=1600^{\circ} \mathrm{C}$

\begin{tabular}{|c|c|c|c|}
\hline$\% F e O$ na escória & $\begin{array}{c}\text { Viscosidade da } \\
\text { escória líquida [Pa.s] }\end{array}$ & $\begin{array}{c}\text { Fração de } \\
\text { fases sólidas }\end{array}$ & $\begin{array}{c}\text { Viscosidade efetiva } \\
\text { da escória [Pa.s] } \\
\text { (Roscoe-Einstein) }\end{array}$ \\
\hline 25 & 0,0300 & 0,327 & 0,0807 \\
\hline 26 & 0,0310 & 0,290 & 0,0729 \\
\hline 27 & 0,0310 & 0,251 & 0,0639 \\
\hline 28 & 0,0310 & 0,212 & 0,0562 \\
\hline 29 & 0,0310 & 0,171 & 0,0495 \\
\hline 30 & 0,0310 & 0,130 & 0,0438 \\
\hline 31 & 0,0310 & 0,087 & 0,0389 \\
\hline 32 & 0,0310 & 0,085 & 0,0387 \\
\hline 33 & 0,0300 & 0,085 & 0,0374 \\
\hline 34 & 0,0290 & 0,085 & 0,0362 \\
\hline 35 & 0,0290 & 0,085 & 0,0362 \\
\hline 36 & 0,0280 & 0,085 & 0,0350 \\
\hline 37 & 0,0280 & 0,085 & 0,0350 \\
\hline 38 & 0,0270 & 0,086 & 0,0338 \\
\hline 39 & 0,0260 & 0,086 & 0,0326 \\
\hline
\end{tabular}

A Tabela 2 apresenta os resultados obtidos por meio do software FactSage 6.4, para o cálculo da viscosidade da escória líquida e fração de sólidos, e os resultados para a

* Contribuição técnica ao $69^{\circ}$ Congresso Anual da ABM - Internacional e ao 14ํㅡㄹ ENEMET - Encontro Nacional de Estudantes de Engenharia Metalúrgica, de Materiais e de Minas, 21 a 25 de julho de 2014, São Paulo, SP, Brasil. 


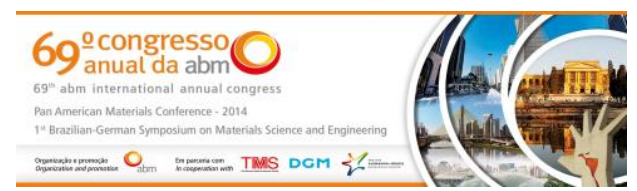

viscosidade efetiva via equação de Roscoe-Einstein. A composição da escória foi limitada, aqui, para o teor de $\mathrm{FeO}$ na faixa entre 25 a 39\% ( $\mathrm{MgO}=10 \%$ e $\mathrm{B}_{2}=2,0$, temperatura $1600^{\circ} \mathrm{C}$ ).

Pode-se observar que a viscosidade da escória líquida varia pouco para o intervalo de composições estudado. Já a viscosidade efetiva é quase três vezes maior do que aquela da líquida para 25\% FeO. A viscosidade efetiva cai continuamente entre $25 \mathrm{e}$ $31 \%$ de $\mathrm{FeO}$, pois a porcentagem da fase $\mathrm{C}_{2} \mathrm{~S}$ formada também cai. A partir de $31 \%$ de $\mathrm{FeO}$ entra-se no campo bifásico contendo as fases líquido mais $\mathrm{MW}$, e o valor da viscosidade efetiva permanece praticamente constante ao longo dos teores crescentes de $\mathrm{FeO}$.

\section{CONCLUSÕES}

O presente trabalho mostra que é possível estudar por meio da termodinâmica computacional alguns tópicos de grande importância para a produção de aço, nominalmente a espumação e a saturação em MgO de escória.

Por meio do sistema quaternário $\mathrm{CaO}_{-} \mathrm{SiO}_{2}-\mathrm{FeO}-\mathrm{MgO}$ (CSFM) foi possível reproduzir (i) composições de saturação das fases sólidas 'MgO' e 'CaO' e (ii) a viscosidade efetiva de um ponto particular deste sistema dado diagrama do tipo ISD.

Nem sempre, contudo, os dados experimentais e os resultados obtidos convergem como foi o caso, por exemplo, da curva de saturação em $\mathrm{MgO}$ em função da basicidade binária.

\section{REFERÊNCIAS}

1 Mourão MB, et al., Introdução à Siderurgia. 2007, São Paulo: Associação Brasileira de Metalurgia, Materiais e Mineração.

2 Pretorius EB, Carlisle RC. Foamy Slag Fundamentals and Their Practical Application to Electric Furnace Steelmaking. In Electric Furnace Conference Proceedings. 1998:

Association for Iron \& Steel Technology (AIST)

3 Morales RD, et al., The slag foaming practice in EAF and its influence on the steelmaking shop productivity. ISIJ International, 1995; 35(9): 1054-1062.

4 Matsuura $\mathrm{H}$, Fruehan RJ. Slag foaming in an electric arc furnace. ISIJ International, 200;. 49(10): p. 1530-1535.

5 Kitamura S, Okohira K. Influence of slag composition and temperature on slag foaming. ISIJ International, 1992. 32(6): 741-746.

6 Hara S, Ogino K. Slag-foaming phenomenon in pyrometallurgical processes. ISIJ International, 1992. 32(1): p. 81-86.

7 Barella S, et al., Critical evaluation of role of viscosity and gas flowrate on slag foaming. Ironmaking and Steelmaking, 2012; 39(6): 463-469.

8 Wu LS, Albertsson GJ. Sichen, D. Modelling of slag foaming. Ironmaking and Steelmaking, 2010; 37(8): 612-619.

9 Kwong J, Bennett JP. Achieving MgO saturated foamy slags in the EAF, In 2001 Electric Furnace Conference Proceedings. 2001, Association for Iron \& Steel Technology: Warrendale. p. 277.

10 Hong L, Hirasawa M, Sano M. Behavior of slag foaming with reduction of iron oxide in molten slags by graphite. ISIJ International, 1998; 38(12): 1339-1345.

$11 \mathrm{Kim} \mathrm{HS}$, Min DJ, Park JH. Foaming behavior of $\mathrm{CaO}-\mathrm{SiO}_{2}-\mathrm{FeO}-\mathrm{MgO}$ sat. $-\mathrm{X}\left(\mathrm{X}=\mathrm{Al}_{2} \mathrm{O}_{3}\right.$, $\mathrm{MnO}, \mathrm{P}_{2} \mathrm{O}_{5}$, and $\mathrm{CaF}_{2}$ ) slags at high temperatures. ISIJ International, 2001. 41(4): 317324.

12 Bale CW, et al., FactSage termochemical software and databases. Calphad, 2002. 26(2): 189-228.

\footnotetext{
* Contribuição técnica ao $69^{\circ}$ Congresso Anual da ABM - Internacional e ao 14ํㅡㄹ ENEMET - Encontro Nacional de Estudantes de Engenharia Metalúrgica, de Materiais e de Minas, 21 a 25 de julho de 2014, São Paulo, SP, Brasil.
} 


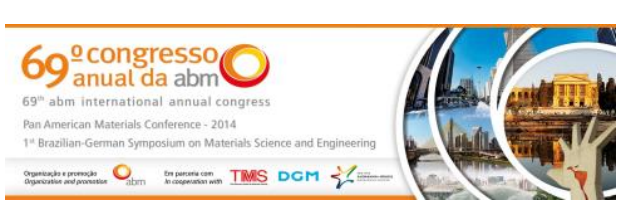

13 Allibert M, Gaye H, et al. Slag Atlas. 2. ed. Düsseldorf: Verlag Stahleisen GmbH, 1995.

14 Wu P, Eriksson G, Pelton AD. Critical Evaluation and Optimization of the Thermodynamic Properties and Phase Diagrams of the $\mathrm{CaO}-\mathrm{FeO}, \mathrm{CaO}-\mathrm{MgO}, \mathrm{CaO}-$ $\mathrm{MnO}$, FeO-MgO, FeO-MnO and MgO-MnO Systems, J. Am. Ceram. Soc., 1993. 76: 2065-2075.

15 Eriksson G, Wu P, Blander M, Pelton AD. Critical Evaluation and Optimization of the Thermodynamic Properties and Phase Diagrams of the $\mathrm{MnO}-\mathrm{SiO}_{2}$ and $\mathrm{CaO}-\mathrm{SiO}_{2}$ Systems, Canad. Met. Quart., 1994.33: 13-22

16 Decterov S, Jung I-H, Pelton,AD., Thermodynamic Modeling of the FeO-Fe2O3-MgOSiO2 System, J. Am. Ceramic Soc., 2002; 85: 2903-2910.

17 Decterov S, Jung I-H, Jak E, Kang Y-B, Hayes $\mathrm{P}$, Pelton AD. Thermodynamic Modeling of the $\mathrm{Al}_{2} \mathrm{O}_{3}-\mathrm{CaO}-\mathrm{CoO}-\mathrm{CrO}-\mathrm{Cr}_{2} \mathrm{O}_{3}-\mathrm{FeO}-\mathrm{Fe}_{2} \mathrm{O}_{3}-\mathrm{MgO}-\mathrm{MnO}-\mathrm{NiO}_{2}-\mathrm{SiO}_{2}-\mathrm{S}$ System and Applications in Ferrous Process Metallurgy, Proceed. VII Intern. Conference on Molten Slags, Fluxes and Salts, South African Inst. Mining \& Metallurgy, Johannesburg, 2004: p. 839-849,.

18 Jung I-H, Decterov S, Pelton AD. Thermodynamic Modeling of the Fe-Mg-O system, J. Phys. Chem. Solids, 2004; 65: 1683-1695.

19 Jung I-H, Decterov, S., Pelton, A.D. Thermodynamic Modeling of the $\mathrm{FeO}-\mathrm{Fe}_{2} \mathrm{O}_{3}-\mathrm{MgO}-$ $\mathrm{SiO}_{2}$ System, Metall. and Mat. Trans. B., 2004. 35B: 877-889.

20 Jung I-H, Decterov S, Pelton AD. Thermodynamic Modeling of the $\mathrm{CaO}-\mathrm{MgO}-\mathrm{SiO}_{2}$ System, J. Eur. Ceram. Soc., 2005; 25: 313-333.

21 Jak E, Hayes P, Pelton A, Decterov S. Thermodynamic optimisation of the $\mathrm{FeO}-\mathrm{Fe}_{2} \mathrm{O}_{3}$, $\mathrm{SiO}_{2}$ (Fe-O-Si) system with FactSage", Intern. J. Mat. Res., 2007. 9: 847-54 ().

22 Trömmel G, Koch K, Fix W, Grobkurth N. Archiv für das Eisenhüttenwesen, 1969; 40(12): p. 969-978.

23 Görl E, Klages R, Scheel R, Trömmel G. Archiv für das Eisenhüttenwesen, 1969. 40(12): p. 959-967.

24 Bennett J, Kwong K-S. Ironmaking and Steelmaking. 2010;37(7):529-535.

\footnotetext{
* Contribuição técnica ao 69ำ Congresso Anual da ABM - Internacional e ao 14ํㅡㄹ ENEMET - Encontro Nacional de Estudantes de Engenharia Metalúrgica, de Materiais e de Minas, 21 a 25 de julho de 2014, São Paulo, SP, Brasil.
} 